Anaesthesist 2018 67:553-554

https://doi.org/10.1007/s00101-018-0471-0

Online publiziert: 19. Juli 2018

(c) Springer Medizin Verlag GmbH, ein Teil von Springer Nature 2018

CrossMark

\section{T. Kammerer}

Klinik für Anaesthesiologie, Ludwig-Maximilians-Universität München, München, Deutschland

\title{
Atemwegstrennung und Einlungenventilation
}

\section{Eine besondere Herausforderung für den Anästhesisten}

de des 19. Jahrhunderts. wurden erste Techniken hierzu entwickelt, die Anfang des letzten Jahrhunderts, zunächst bei freiwilligen Probanden, erstmals zur Anwendung kamen [5]. Den Durchbruch brachte 1949 der erste von Eric Carlens in Stockholm entwickelte Doppellumentubus, der vom Aufbau her unseren heute verfügbaren Doppellumentuben am nächsten kommt [1]. Seit damals hat sich diese Technik weltweit etabliert und gilt heute als „Goldstandard“ der Einlungenventilation [2]. Durch eine enge Zusammenarbeit von Ärzten und Industrie stehen mittlerweile zahlreiche „devices“ zur Verfügung, die eine Lungenseparation in beinahe jedem Lebensalter ermöglichen. Der Arbeit von Meggiolaro et al. geht darauf detailliert ein und stellt auch die neuerdings verfügbaren Doppellumentuben mit integrierter Videooptik vor [6]. In einer aktuellen randomisierten Studie konnte bereits gezeigt werden, dass die Notwendigkeit einer Fiberoptik zur initialen Positionierung und intraoperativen Neupositionierung durch die Verwendung eines Videodoppellumentubus im Vergleich zum konventionellen Doppellumentubus signifikant reduziert werden kann [3]. Man darf gespannt sein, welchen Stellenwert dieser Tubus in $\mathrm{Zu}$ kunft haben wird.

Auch wenn der Doppellumentubus die bei Weitem häufigste Methode zur Lungenseparation darstellt, so gibt es doch immer wieder Situationen, in denen er nicht zur Anwendung kommen kann. In diesen Fällen bieten Endobronchialblocker häufig die einzige Alternative. Die wichtigsten Modelle werden im aktuellen Übersichtsartikel ebenfalls übersichtlich erläutert. Leider werden Bronchusblocker in der täglichen Routine nur selten genutzt, sodass die Handhabung, z. B. in Notfallsituationen, oft Schwierigkeiten bereitet. Es muss daher gefordert werden, diese im Rahmen der täglichen Arbeit häufiger anzuwenden.

\section{Praktische Übungen essenziell}

Eine wichtige Voraussetzung, um Techniken zu erlernen und Schwierigkeiten der Lungenseparation adäquat zu behandeln, ist der Besuch von Schulungen und Workshops, wie sie z.B. der Arbeitskreis „Anästhesie in der Thoraxchirurgie“ der DGAI regelmäßig im Rahmen des Deutschen Anästhesiekongresses (DAC) oder des Hauptstadtkongresses der DGAI (HAI) anbietet. Egal, ob Doppellumentuben oder Endobronchialblocker zum Einsatz kommen; die Handhabung der Fiberoptik muss in der Thoraxanästhesie beherrscht werden. Grundlagenkenntnisse der Bronchoskopie sind daher essenziell und haben ihren Nutzen auch im zweiten Standbein unseres Fachgebietes, der Intensivmedizin. Auch hier bieten Workshops und Simulatoren eine gute Basis für die Anwendung in der täglichen Praxis.

Ebenso wichtig wie praktische Fertigkeiten ist aber auch ein fundiertes Wissen der pathophysiologischen Veränderungen während der Lungenseparation. Die Interaktion zwischen Ventilation und Hämodynamik ist auch für erfahrene Kolleginnen und Kollegen immer wieder beeindruckend! Spannend wird es insbesondere dann, wenn patientenbezogene, chirurgische und anästhesioon ist länger, als man denkt: Bereits En- 
logische Faktoren die physiologischen Reaktionen innerhalb der Lunge und des kleinen Kreislaufs beeinträchtigen und beispielsweise eine Hypoxämie oder Rechtsherzproblematik adäquat behandelt werden muss [7].

\section{Offenen Fragen}

Trotz zahlreicher randomisierter Untersuchungen im Bereich der Thoraxanästhesie gibt es nach wie vor brennende Fragen, die geklärt werden müssen. So wird z. B. die aktuell laufende multizentrische PROTHOR-Studie wichtige Erkenntnisse zur Bedeutung von PEEP und Rekrutierungsmanövern während der Einlungenventilation erbringen. Ebenso bedeutsam sind Initiativen, die der Qualitätssicherung dienen und einen „standard of care" in der Thoraxanästhesie und Thoraxchirurgie definieren. Hierzu wird u.a. das Deutsche Thoraxregister, eine Datenbank in Kooperation der Deutschen Gesellschaft für Anästhesiologie und Intensivmedizin (DGAI) mit der Deutschen Gesellschaft für Thoraxchirurgie (DGT), in den nächsten Jahren wichtige Informationen liefern können. Insgesamt ist und bleibt die Thoraxanästhesie eines unserer abwechslungsreichsten Tätigkeitsfelder, und man darf sicher sein, dass dies auch in der Zukunft so sein wird.

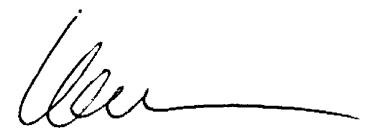

T. Kammerer

\section{Korrespondenzadresse}

\section{Dr. T. Kammerer}

Klinik für Anaesthesiologie, Ludwig-

Maximilians-Universität München

Marchioninistr. 15, 81377 München,

Deutschland

tobias.kammerer@med.uni-muenchen.de

Interessenkonflikt. T. Kammerer gibt an, dass kein Interessenkonflikt besteht.

\section{Literatur}

1. Carlens E (1949) A new flexible double-lumen catheter for bronchospirometry. J Thorac Surg 18:742-746

2. Defosse J, Schieren M, Bohmer A et al (2016) Deutschlandweite Umfrage zur Thoraxanasthesie. Anaesthesist 65:449-457

3. Heir JS, Guo SL, Purugganan R et al (2018) A Randomized Controlled Study of the Use of Video Double-Lumen Endobronchial Tubes Versus Double-Lumen Endobronchial Tubes in Thoracic Surgery. J Cardiothorac Vasc Anesth 32:267-274

4. Kammerer T, Speck E, Von Dossow V (2016) Anästhesie in der Thoraxchirurgie. Anaesthesist 65:397-412

5. Mcgrath B, Tennuci C, Lee G (2017) The History of One-Lung Anesthesia and the Double-Lumen Tube. J Anesth Hist 3:76-86

6. Meggiolaro KM, Wulf $\mathrm{H}$, Feldmann C et al (2018) Atemwegsmanagement zur Seitentrennung der Lunge bei thorakalen Eingriffen. Anaesthesist. https://doi.org/10.1007/s00101-018-0470-1

7. Schafer B, Greim CA (2018) Akute perioperative Rechtsherzinsuffizienz: Diagnostik und Therapie. Anaesthesist 67:61-78

\section{Perioperative Medizin}

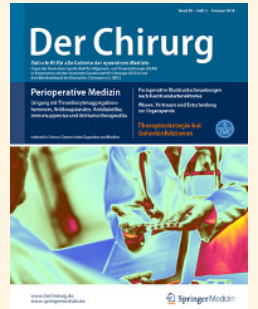

Eine steigende Zahl zu operierender Patienten muss ein oder mehrere Medikamente einnehmen. Dies stellt den betreuenden Arzt vor die Frage,

ob diese ohne Unterbrechung von der Zeit vor bis nach der Operation weitergeführt werden können, ob damit kurz oder langfristig pausiert werden muss oder ob ein "bridging" erforderlich ist.

Wertvolle Handlungsempfehlungen aus Sicht der Autoren bildet das Leitthema in Der Chirurg 02/2018 ab. Es bietet eine praxisrelevante Übersicht über den perioperativen Umgang mit

- Thrombozytenaggregationshemmern

- Antikoagulation

- Antidiabetika

- Oralen Tumortherapeutika

- Immunsuppressiver Therapie.

\section{Suchen Sie noch mehr zum Thema?} Mit e.Med - den maßgeschneiderten Fortbildungsabos von Springer Medizin - haben Sie Zugriff auf alle Inhalte von SpringerMedizin.de. Sie können schnell und komfortabel in den für Sie relevanten Zeitschriften recherchieren und auf alle Inhalte im Volltext zugreifen.

Weitere Infos zu e.Med finden Sie auf springermedizin.de unter "Abos" 